\title{
RECAI
}

RECAI Revista de Estudios en Contaduría,

Administración e Informática

ISSN: 2007-5278

danielarturoc@yahoo.com.mx

Universidad Autónoma del Estado de México

México

\section{Adopción de la responsabilidad social empresarial en una empresa manufacturera de Mexicali, Baja California}

Gómez Córdova, Bernardette Eunice; Vela Reyna, Juan Benito

Adopción de la responsabilidad social empresarial en una empresa manufacturera de Mexicali, Baja California

RECAI Revista de Estudios en Contaduría, Administración e Informática, vol. 9, núm. 24, 2020

Universidad Autónoma del Estado de México, México

Copyright 2019 Universidad Autónoma del Estado de México

Esta obra está bajo una Licencia Creative Commons Atribución-NoComercial-SinDerivar 4.0 Internacional. 


\section{Adopción de la responsabilidad social empresarial en una empresa manufacturera de Mexicali, Baja California}

The adoption of corporate social responsibility in a manufacturing company in Mexicali, Baja California

Bernardette Eunice Gómez Córdova

Universidad Autónoma de Baja California (UABC),

México

eunice.gomez@uabc.edu.mx

Juan Benito Vela Reyna

Universidad Autónoma de Baja California (UABC),

México

jbenitovela@uabc.edu.mx

Recepción: 28 Agosto 2019

Aprobación: 25 Noviembre 2019

\section{Resumen:}

El presente estudio tiene como objetivo identificar y reconocer el efecto que genera el adoptar prácticas de responsabilidad social empresarial (RSE) en los trabajadores del área de producción de una empresa del sector de manufactura en la Ciudad de Mexicali, Baja California. Para ello, se analizó primeramente la literatura para identificar y validar este comportamiento mediante un modelo ya propuesto, que además incluyera un instrumento que permitiera explicar la relación existente en las variables referentes al aspecto del cuidado de la salud, la seguridad y las condiciones de trabajo, el desarrollo profesional y la empleabilidad, y el alcance que pueda llegar a tener en el ámbito familiar de los colaboradores. La muestra fue trazada considerando los tres turnos de operatividad laboral de la empresa. Los principales hallazgos indican que los trabajadores detectan positivamente la aplicación de prácticas de RSE. Los empleados, además, reconocieron que la empresa realiza actividades para el desarrollo dentro y fuera de su área de trabajo; lo que les proporciona un sentido de pertenencia y calidad de vida.

PALABras CLAVE: responsabilidad social empresarial, capital humano, calidad de vida.

\section{ABstract:}

The purpose of this study is to identify and recognize the effect generated by the adoption of corporate social responsibility (CSR) practices on the well-being of the production-area workers of a manufacturing-sector company in the City of Mexicali, Baja California. To do so, we first review the relevant literature to identify and validate such behavior through an existing model that includes an instrument that would help explain the relationship among several variables referring to health care, safety and working conditions, professional development and employability, and the scope that may have in the family of employees. The sample was drawn considering the three shifts of labor operability that the company has. This study's main findings indicate that workers positively detect the application of CSR practices. Also, the workers acknowledged that the company undertakes activities that promote their development in and out of their working areas, which, in turn, provides them with a sense of belongingness and quality of life.

KEYWORDS: corporate social responsibility, human capital, quality of life.

\section{INTRODUCCIÓN}

Asumir una posición frente a la responsabilidad social influye sobre diversos aspectos de la gestión organizacional y la gestión de lo humano resulta ser uno de los elementos críticos al respecto, pocos estudios se han enfocado en considerar este aspecto con relación a la Responsabilidad Social Empresarial, de manera habitual la RSE exige a la empresa el cumplimiento de todas las responsabilidades económicas, legales, éticas y filantrópicas. 
El Pacto Mundial, se propone aprovechar la fuerza de la acción colectiva para fomentar la responsabilidad cívica de las empresas de modo que éstas puedan contribuir a la solución de los retos que plantea la globalización. en este sentido el Pacto Mundial "No define el concepto de RSE como tal; sin embargo consideran que las prácticas empresariales basadas en principios universales contribuyen a la construcción de un mercado global más estable, equitativo e incluyente que fomentan sociedades más prósperas". Dentro de sus objetivos y funciones destaca integrar los cambios necesarios en las operaciones, de tal manera que el Pacto Mundial y sus principios sean parte de la gestión, la estrategia, la cultura y el día a día de la actividad empresarial, alrededor de 4 ejes temáticos: derechos humanos, condiciones laborales, medio ambiente y anticorrupción.

La RSE ha tomado fuerza y protagonismo en muchas de las empresas en la actualidad, sin importar su tamaño o giró, como resultado de una búsqueda de consolidarse como empresas que implementan y proveen el bienestar social (capital humano) dentro de las prácticas de RSE que implementan; partiendo de lo anterior, Peláez, García y Azuero (2014) toman en cuenta tres situaciones mínimas que se deben propiciar en la organización para que tanto la RSE como el área encargada de la gestión humana interna generen valor a la empresa, Debe crearse un compromiso de la dirección o gerencia con los trabajadores y con la RSE, el gerente del área de gestión humana debe contar con una considerable formación gerencial e interactuar con la cabeza directiva de la empresa, respondiendo y actuando siempre en beneficio de los trabajadores, así mismo la gestión humana y la RSE deben estar encaminadas y alineadas estratégicamente con la empresa, atendidas por estrategias precisas que deben ser identificadas y reconocidas por todos los integrantes de la organización, con el objetivo de no limitar las acciones, ni de gestión humana, ni de la RSE.

El estudio sobre la RSE es un concepto ampliamente debatido y de gran trayectoria histórica a lo largo del siglo pasado e inicios del presente, en el cual ha retomado gran auge y desarrollo en el ámbito empresarial, (Romo, 2016), a pesar de que este tipo de estrategias han marcado una tendencia en adopción, sigue siendo un número pequeño de empresas que buscan con interés adoptar y mantener este tipo de certificaciones según resultados mostrados en el IX Encuentro Latinoamericano de Empresas Socialmente Responsables (ESR), en dónde 1,364 alcanzaron el distintivo ESR-2016 de un total de 1,498 empresas inscritas en el proceso

El primer aspecto refiere a la RSE como orientación de acciones de quienes trabajan el área de gestión humana, el cual comprende de estrategias, políticas, transparencia de procesos, rendición de cuentas, valores y principios, el segundo, la RSE en las acciones de la gestión humana, enfocado al logro de la satisfacción de necesidades de los trabajadores, tercer aspecto, la RSE como evaluación de las acciones de gestión humana, incentivando la colaboración en áreas otorgadas y creados por la organización para difundir e implementar la RSE y en el cuarto aspecto, la RSE en el aporte de gestión humana, según el reconocimiento (sea positivo o negativo), así corresponderá la influencia del trabajador y así mismo se verá reflejado en resultados como el desempeño y sobre todo en lo más importante su calidad de vida laboral y personal.

Por lo anterior el principal reto recaen en poder valorar las estrategias implementadas para que la empresa logre mantener su certificación de RSE, e identificar si existen resultados positivos en el desempeño del trabajador perteneciente al área de producción de una empresa manufacturera situada en la ciudad de Mexicali, Baja California; para lograr esto se pretende realizar una búsqueda e identificar las principales teorías que expliquen de la RSE, y que conlleven a una clara apreciación del personal sobre su cuidado de la salud, seguridad y condiciones de trabajo, el desarrollo profesional y empleabilidad y el alcance que pueda llegar a tener en el ámbito familiar de los colaboradores.

\section{REVISIÓN LITERARIA}

Las empresas cuentan con elementos básicos en su estructura como son; elementos humanos, aspiraciones, realizaciones, bienes materiales, su capacidad técnica, capacidad financiera, la producción, transformación y/o prestación de servicios, para llegar a lograr la satisfacción de necesidades y deseos del consumidor 
final y mercado meta. Para efectos de este estudio, nos enfocamos a la recopilación de datos, información, investigación, análisis y desarrollo de la RSE desde adentro de una empresa que cuenta con el distintivo, por llevar a cabo estrategias y acciones dirigidas hacia el desarrollo y atención a sus trabajadores y familias, para lo cual se tomaron en consideración algunas propuestas realizadas por distintos autores que definen la RSE desde la visión interna de la propia empresas, en este caso el área de gestión humana.

Iniciando con Ena y Delgado, (2012), propone que "La responsabilidad social interna se entiende como la parte de la RSE que gestiona de manera social, sostenible, ética, humanista y solidaria los recursos humanos de una empresa" pp 58.

Por otro lado en la búsqueda de fomentar un marco europeo para la responsabilidad social de las empresas la Comisión Europea en su Libro verde, (2001) menciona que la dimensión interna es aquella que viene delimitada por las obligaciones de responsabilidad de la empresa hacia sus trabajadores y hacia sus procesos de producción. En este ámbito interno el Libro Verde sitúa cuatro áreas de responsabilidad: la gestión de recursos humanos; las cuestiones de salud y seguridad, en el lugar de trabajo; los procesos de adaptación al cambio; y la gestión del impacto ambiental y de los recursos naturales. (Error 3: La referencia debe estar ligada) (Error 4: El tipo de referencia es un elemento obligatorio) (Error 5: No existe una URL relacionada)

La Comisión Europea, (2001) "Gestión de Recursos Humanos: Es uno de los desafíos más importantes a los que se enfrentan las empresas es atraer a trabajadores calificados y lograr que permanezcan a su servicio. Las medidas pertinentes podrían incluir el aprendizaje permanente, responsabilidad de los trabajadores, mejora de la información en la empresa, mayor equilibrio entre trabajo, familia y ocio, mayor diversidad de recursos humanos, igualdad de retribución y de perspectivas profesionales para las mujeres, participación en los beneficios de la empresa, la consideración de la capacidad de inserción profesional y la seguridad en el lugar de trabajo".

Jaramillo, (2011), La RSE en su dimensión interna, sobrepasa el cumplimiento de las imposiciones legales en materia de recursos humanos, se precisa que sea entendida por la gestión de recursos humanos como la incorporación espontánea de un conjunto de políticas y acciones que resulten en un aporte al bienestar sostenido de sus trabajadores y por ende, a su entorno social, como una forma de obtener ventaja competitiva. pp. 170

Tomando como base y referencia a los diferentes autores anteriormente mencionados, destacamos que toda aquella actividad o práctica que vaya enfocada al desarrollo integral del trabajador, como es educación, nuevas habilidades de trabajo, cuidado en la salud y seguridad, tales como las condiciones de trabajo, seguimiento al ambiente laboral y lo referente a actividades dirigidas y/o de beneficio a las familias como eventos de recreación, desarrollo de estudios a hijos y cónyuges e involucramiento de familiares a la empresa en eventos especiales, ayudarán al logro de una calidad de vida, generará bienestar y compromiso de un trabajador dentro de cualquier organización donde se desempeñe, además de aportarles un valor agregado a los trabajadores y la empresa.

Para efectos de este estudio los objetivos fueron identificar y reconocer el efecto que generan el implementar las prácticas de RSE como caso de estudio en los trabajadores del área de producción de una empresa del sector manufactura situada en la Ciudad de Mexicali, Baja California, intentando poder validar mediante la aplicación de un instrumento que contempla tres dimensiones que resultan de mayor importancia para la empresa, en primer lugar evaluando los aspectos del cuidado de la salud, seguridad y condiciones de trabajo, permitiendo testificar el cumplimiento y orientación de acciones en un mayor número de estas para lograr cumplir y garantizar esto en el personal, en segundo lugar su desarrollo profesional y empleabilidad, teniendo como resultado en la empresa una calidad de vida laboral en sus trabajadores a través de estrategias implementadas hasta el momento sobre la RSE, Peláez-León et al.(2014) explica la relación de la RSE y la gestión humana a partir de las variables señaladas en este mismo párrafo.

Considerando las prácticas que se llevan en la empresa en dónde se realizó este caso, se adaptó el modelo de los autores Peláez-León et al. (2014) se logró formular un esquema presentado en la Figura 1, partiendo 
de poder comprobar si resulta factible poder alcanzarse la RSE por parte de la empresa cumpliendo con los siguientes enfoques, orientándose en primer lugar en el desarrollo profesional y empleabilidad mediante la capacitación continua, otorgamiento de reconocimientos y/o bonificaciones individuales y/o colectivas por el desempeño de los trabajadores, la entrega de becas o auxilio y/o asistencia para la realización de estudios que permiten lograr la superación profesional, y en la segunda el cuidado de la salud, seguridad y condiciones de trabajo logrando cumplir con programas de inducción y entrenamiento, realizando investigaciones sobre el ambiente laboral, realizando supervisiones sobre las condiciones de trabajo para mejorar la calidad de vida laboral e implementando en zonas aledañas a las áreas de trabajo espacios de recreación de convivencias y agregando a manera de propuesta una tercera dimensión que proyecta un alcance que pueda llegar a tener la RSE en el ámbito familiar de los colaboradores, generando un balance trabajo-familia y como resultado una calidad de vida, realizando en la empresa programas de bienestar dirigidos a la salud, vivienda, deporte, recreación, préstamos, auxilios y/o becas para la educación de hijos de trabajadores, eventos festivos de integración con familiares de trabajadores como día del niño, navidad, así como eventos de reconocimiento al compromiso de los trabajadores en los cuales invitan a ser parte a los familiares, talleres de capacitación para cónyuges y/o hijos y jornadas de vacunación y salud para familias del trabajador.

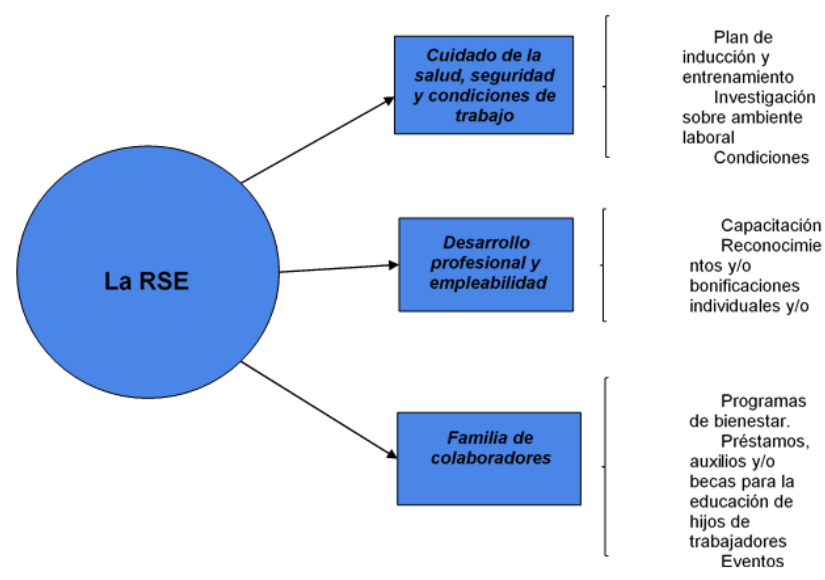

FIGURA 1.

Esquema Instrumento Aplicado

Fuente: Adaptación al modelo explicativo desde una perspectiva estratégica, Peláez León, García y Azuero (2014)

\section{MÉTodo}

El presente estudio es de tipo cuantitativo debido al instrumento de análisis que se utilizó encuesta en escala de Likert, aplicado a 150 trabajadores del área de producción de una empresa del sector manufactura, dedicada a la manufactura de autopartes: dispositivos eléctricos para la activación de bolsas de aire, ubicada en la Ciudad de Mexicali, B.C., el instrumento fue aplicado a los tres turnos con los que cuenta la planta y respectivamente según la cantidad de trabajadores por turno.

Es de tipo cualitativa por qué desde el inicio de la investigación y recolección de información se acudió a la empresa para indagar en los trabajos y prácticas que llevaban a cabo dentro del tema de RSE, se recabó información importante dentro de los medios de difusión digital con los que cuenta la empresa que ayudaron a constatar y/o cuestionar las actividades o prácticas que realizan hacia sus trabajadores. Además el instrumento aplicado cuenta con un apartado de pregunta abierta la cual aportó de gran manera conocer el sentir y expectativas del trabajador hacia la empresa. 
En este mismo sentido fue de gran apoyo para recabar información, detalles de comportamiento, observación, etc, el asistir personalmente a la aplicación del instrumento a los tres turnos de la empresa, al primer turno se aplicaron 78 encuestas, segundo turno 51 y tercer turno 21.

Con el objetivo de conocer, analizar y demostrar el sentir y respuesta de los trabajadores en relación con las prácticas de RSE que implementa la empresa, basado en el modelo mencionado al inicio de este apartado, se creó el instrumento a aplicar, la estructura del mismo consta de 12 preguntas en escala de likert dividido en 3 aspectos a evaluar en relación con la RSE contando con 4 preguntas cada apartado, al final de cada una se indica una pregunta en formato abierta.

El primer apartado indicado en el instrumento hace referencia a prácticas dirigidas al desarrollo profesional y empleabilidad hacia el trabajador, el segundo al cuidado de la salud, seguridad y condiciones de trabajo, y el tercer apartado al de familias de los colaboradores.

Para el análisis de los datos obtenidos mediante la aplicación del instrumento se utilizó un modelo de ecuaciones estructurales basado en mínimos cuadrados parciales (SmartPLS, por sus siglas en inglés), ya que permite examinar simultáneamente una serie de relaciones de dependencia entre variables independientes y dependientes.

\section{Resultados}

Para valorar la hipótesis en la relación de la adopción de la responsabilidad social empresarial con los resultados en el desarrollo profesional y un alcance en el ámbito familiar del trabajador del área de producción se realizó el análisis al total de la muestra que contemplaba 150 encuestas, permitiendo obtener las cargas factoriales de las variables observables. 
TABLA 1.

Consistencia Interna de Cargas Factoriales (Total de encuestados)

\begin{tabular}{|c|c|c|c|}
\hline $\begin{array}{l}\text { Prácticas de Responsabilidad Social } \\
\text { Empresarial }\end{array}$ & & & \\
\hline & FL & CR & $\mathrm{AC}$ \\
\hline $\begin{array}{l}\text { VL2. DESARROLLO PROFESIONAL Y } \\
\text { EMPLEABILIDAD }\end{array}$ & & 0.818 & 0.705 \\
\hline Programa de capacitación técnica para el trabajo & 0.732 & & \\
\hline $\begin{array}{l}\text { Reconocimiento y'o beneficios individuales y/o } \\
\text { generales, según el desempeño }\end{array}$ & 0.694 & & \\
\hline $\begin{array}{l}\text { Becas o ayudas de estudio (educación básica, } \\
\text { media, complementarios, superiores o } \\
\text { posgrados) }\end{array}$ & 0.655 & & \\
\hline Capacitación en temas de interés general & 0.824 & & \\
\hline $\begin{array}{l}\text { VL1. CUIDADO DE LA SALUD, SEGURIDAD Y } \\
\text { CONDICIONES DE TRABAJO }\end{array}$ & & 0.840 & 0.744 \\
\hline $\begin{array}{l}\text { Plan de inducción y entrenamiento dirigido al } \\
\text { trabajador }\end{array}$ & 0.677 & & \\
\hline Investigaciones sobre el ambiente laboral & 0.765 & & \\
\hline $\begin{array}{l}\text { Condiciones de trabajo que contribuyen al } \\
\text { mejoramiento de la calidad de vida laboral }\end{array}$ & 0.857 & & \\
\hline $\begin{array}{l}\text { Espacios para la diversión y la recreación en la } \\
\text { empresa }\end{array}$ & 0.706 & & \\
\hline VL3. FAMIUIA DE LOS COLABORADORES & & 0835 & 0.751 \\
\hline $\begin{array}{l}\text { Programas de bienestar (vivienda, salud, } \\
\text { deporte, recreación, servicios y/o actividades } \\
\text { sociales) para la familia de los trabajadores }\end{array}$ & 0.817 & & \\
\hline $\begin{array}{l}\text { Eventos feslivos para la integración de las } \\
\text { familias (día del niño, navidad, día de muertos) }\end{array}$ & 0.646 & & \\
\hline Talleres de capacitación para esposo(a) ylo hijos & 0.687 & & \\
\hline $\begin{array}{l}\text { Jornadas de vacunación y salud para las } \\
\text { familias de los trabajadores }\end{array}$ & 0.832 & & \\
\hline
\end{tabular}

Fuente: Creación propia basada en programa Smart PLS. FL= Cargas

Factoriales, $\mathrm{CR}=$ Fiabilidad Compuesta, $\mathrm{AC}=$ Alfa de Cronbach.

En los ítems perteneciente al Desarrollo Profesional y Empleabilidad, Cuidado de la Salud, Seguridad y Condiciones de trabajo y Familia de los Colaboradores presentan en su mayoría valores aceptables con cargas arriba de .07 como proponen los autores Carmines y Zeller (1979), sin embargo algunos caen en valores inferiores entre 0.694 y 0.646 para lo cual se debiera considerar su eliminación sin embargo el autor Barclay (et al., 1995) sugiere la no eliminación del ítems siempre y cuando esté su valor arriba de .05 para efectos de la descomposición del modelo.

Para evaluar la consistencia interna de los indicadores de cada constructo permitiendo determinar la rigurosidad de la variable latente que representa, se determinó la fiabilidad compuestas ( $p$ ) y el Alfa de Cronbach, alcanzando un valor arriba de 07 en cada constructo resultado, totalmente aceptables y apegado a los señalados por los autores Fornell y Larcker (1981).

Después de valorar los resultados referentes al modelo de medida, permitieron evaluar la validez de un modelo a través de la consistencia interna con el Alfa de Cronbach y la fiabilidad compuesta, se determinó la validez convergente (varianza media extraída AVE), la cual nos indica lo diferente qué son los constructos considerados utilizando la propuesta de Fornell y Larcker (1981) que señalan que un constructo estará alto de validez discriminante, cuando la AVE de cada constructo fueron aceptables, además en la correlación se lograron resultaron mayores al inferior y para cada caso igualmente superior con el del lado izquierdo 
TABLA 2

Validez Discriminante del Modelo Teórico al análisis general de encuestados

Tabla 2. Validez Discriminante del Modelo Teórico al análisis general de encuestados

\begin{tabular}{|l|c|c|c|c|}
\hline & $\begin{array}{c}\text { Validez } \\
\text { Discriminante }\end{array}$ & \multicolumn{3}{|c|}{ Correlación de validez discriminante } \\
\hline Constructo & AVE & CSSCT & DPyE & FC \\
\hline CSSCT & 0.569 & 0.754 & & \\
\hline DRyE & 0.532 & 0.686 & 0.729 & 0.750 \\
\hline FC & 0.562 & 0.608 & 0.539 & \\
\hline
\end{tabular}

Fuente: Creación propia basada en el programa Smart PLS

Fuente: Creación propia basada en el programa Smart PLS

Los tres valores afirman que los ítems seleccionados en cada constructo representa de manera adecuada y aceptable al constructo subyacente, afirmando la propuesta de los autores Fornell y Larcker (1981), anteriormente mencionada.

\section{DiscuSIÓN}

En base a la teoría revisada sobre la RSE, sus prácticas dirigidas hacia la calidad de vida laboral y los resultados obtenidos en el instrumento aplicado en este estudio donde se analizó las dimensiones referentes al desarrollo profesional y empleabilidad, cuidado de la salud, seguridad y condiciones de trabajo y familia de los colaboradores, arrojaron resultados aceptables, dando respuesta de qué el fomentar prácticas dirigidas hacia los trabajadores tienen buena aceptación por estos últimos, teniendo presente lo que la empresa realiza en diferentes actividades para contribuir al crecimiento y reconocimiento laboral y alcance al ámbito familiar logrando una calidad de vida laboral y personal.

Algunos hallazgos importantes dentro del estudio es que si bien las prácticas de RSE que tiene la empresa hacia sus trabajadores sí es reconocida, captada y agradecida por los trabajadores, varió un poco la respuesta según el turno de trabajo, como ya se indicó anteriormente, la investigación fue aplicada en particular a los trabajadores del área de producción de una empresa de manufactura con tres horarios laborales, en los turnos matutino y vespertino, mostraron más conocimiento sobre las prácticas de RSE que la empresa 
realiza, experiencia en los eventos y actividades realizadas como parte de las prácticas de RSE e interés en retroalimentar a la empresa en el sentir de ellos como trabajadores para aumentar dichas prácticas dentro de los aspectos evaluados, mientras que en el último turno que se realiza durante la madrugada, el nivel de atención que recibieron de dichas prácticas, se débil, por lo que pudiéramos atribuirlo a que dicho turno es de menos horas de trabajo, el nivel físico no es tan alerta en esos horarios, el interés del trabajador no pudiera ser muy alto, pudiera ser que tenga otra actividad laboral en el día y el que esta empresa le otorgue o no beneficios de desarrollo pudiera ser no importante para él.

De manera general la mayoría de las prácticas evaluadas dentro del instrumento aplicado, son muy bien adoptadas y han tenido buenas experiencias los trabajadores de la empresa, por lo que se da respuesta favorable a la relación de ser una empresa socialmente responsable y realizar prácticas hacia el desarrollo de los trabajadores y que conlleve a otorgar una calidad de vida laboral con un alcance hasta el ámbito familiar.

\section{Conclusiones y Recomendaciones}

A través del instrumento aplicado y validado se pudo constatar que los trabajadores del área de producción perciben de manera positiva las prácticas que la empresa realiza dirigidos al desarrollo personal y familiar, de manera que es un resultado positivo y de valor para la empresa.

Se recomienda realizar todavía más difusión a las prácticas, haciendo del conocimiento a los trabajadores sobre la implementación de la RSE, su objetivo y ventajas para que sea todavía mayor y positiva la respuesta de la gente, así como realizar un poco más de actividades dirigidas a los familiares esto solamente por ser la dimensión que obtuvo datos un poco más débil.

La empresa que en la actualidad busque un serio enfoque hacia la responsabilidad social, llevando a cabo prácticas creativas, innovadoras, de desarrollo y crecimiento humano a sus trabajadores, como se demostró en el caso de la empresa que se trabajó en este estudio, garantizará una satisfacción y calidad de vida de ellos, logrando hasta un alcance al ámbito familiar y qué mejor todavía que la misma empresa sea reconocida por ese valor añadido.

La RSE es un reto importante y necesario que tomen hoy en día las organizaciones no importa su giro o tamaño, debido a que deja ver que la organización se preocupa y pone atención a mejorar y desarrollar sus procesos hacia su comunidad de trabajadores, en lo económico, ambiental y social, llegando a ser de ejemplo para la comunidad que la rodea.

Ser una empresa socialmente responsable es importante hoy más que nunca escuchando el entorno interno y externo, se tiene que estar preparado para ayudar a colaborar con la sociedad y ya no solo ver los beneficios económicos, las personas hoy en día tienen un poder individual, fruto de la tecnología, del alcance de las plataformas digitales, hoy el mundo es transparente, hoy no se puede ocultar nada.

Las organizaciones que cuenten con prácticas dirigidas hacia la RSE enfocadas al crecimiento de su recurso humano, irán más allá de generar utilidades, sino que aportaran un valor agregado a su gente.

La tendencia y el mundo globalizado de estos tiempos va en camino a crear un valor significativo a las personas en el entorno donde se desarrollan, no olvidemos que el recurso más importante con el que puede contar la empresa es con su gente, desde siempre se ha buscado retener al personal, bajar índices de rotación de personal, lograr un ambiente organizacional óptimo, que mejor que alcanzar todo lo anterior y mucho más a través de procesos, actividades, prácticas que vayan más allá de normativas, reglamentos, políticas y concedan un valor significativo a las personas, su desarrollo y su ámbito familiar, hasta lograr una calidad de vida.

\section{REFERENCIAS}

Barclay, D., R. Thompson, y C. Higgins. (1995). The Partial Least Squares (PLS) Approach to Causal Modeling: Personal Computer Adoption and Use an Illustration, Technology Studies (2) 2, 285-309. 
RECAi Revista de Estudios en Contaduría, Administración e Informática, 020, 9(24), Enero-Abril, I...

Carmines, E. y Zeller, R. (1979). Reliability and Validity Assessment. N. 07-017, Sage University Paper Series on Quantitative Applications the Social Sciences. Beverly Hills, CA: Sage.

Comisión Europea (2001). Libro verde: Fomentar un Marco Europeo para la Responsabilidad Social de las Empresas. Bruselas: Unión Europea.

Delgado González, S., \& Ena Ventura, B. (2012). Recursos Humanos y Responsabilidad Social Corporativa, 58

Fornell, C.; Larcker, D.F. (1981). Evaluating structural equation models with unobservable variables and measurement error, Journal of Marketing Research, 18, 39-50.

Jaramillo O. 2011. La dimensión de la responsabilidad social en las micro, pequeñas y medianas empresas del Programa EXPOPYME de la Universidad del Norte. Pensamiento y Gestión. 31, 167- 195.

Peláez et al., (2014), J. Peláez, M. García, A. Azuero, Relación entre la Responsabilidad Social Empresarial y la Gestión Humana: propuesta de un modelo explicativo desde una perspectiva estratégica. Documento de trabajo, Universidad del Valle - Cali, Colombia

Romo Ana María (2016), Responsabilidad Social Empresarial y su evolución en México, medio ambiente y sociedad, 5to. Congreso Nacional de Ciencias Sociales, La Agenda Emergente de las Ciencias Sociales.

Copyright 2019 Universidad Autónoma del Estado de México

CC BY-NC-ND 